\title{
Hunting Sodium Dendrites in NASICON-Based Solid-State Electrolytes
}

\author{
Qiangqiang Zhang ${ }^{D},{ }^{1,2}$ Yaxiang Lu $\mathbb{D}^{1},{ }^{1,3}$ Weichang Guo, ${ }^{4}$ Yuanjun Shao, ${ }^{1,2}$ Lilu Liu, ${ }^{1,2}$ \\ Jiaze Lu, ${ }^{1,2}$ Xiaohui Rong, ${ }^{1,2}$ Xiaogang Han ${ }^{(D)}{ }^{4}$ Hong Li, ${ }^{1,2,3}$ Liquan Chen, ${ }^{1,2,3}$ \\ and Yong-Sheng $\mathrm{Hu} \mathbb{1 D}^{1,2,3}$
}

\begin{abstract}
${ }^{1}$ Key Laboratory for Renewable Energy, Beijing Key Laboratory for New Energy Materials and Devices, Beijing National Laboratory for Condensed Matter Physics, Institute of Physics, Chinese Academy of Sciences, Beijing 100190, China

${ }^{2}$ Center of Materials Science and Optoelectronics Engineering, University of Chinese Academy of Sciences, Beijing 100049, China

${ }^{3}$ Yangtze River Delta Physics Research Center Co. Ltd., Liyang 213300, China

${ }^{4}$ State Key Laboratory of Electrical Insulation and Power Equipment, School of Electrical Engineering, Xi'an Jiaotong University, Xi'an, Shaanxi 710049, China
\end{abstract}

Correspondence should be addressed to Yaxiang Lu; yxlu@iphy.ac.cn, Xiaogang Han; xiaogang.han@xjtu.edu.cn, and Yong-Sheng Hu; yshu@iphy.ac.cn

Received 28 February 2021; Accepted 21 April 2021; Published 22 May 2021

Copyright (c) 2021 Qiangqiang Zhang et al. Exclusive Licensee Beijing Institute of Technology Press. Distributed under a Creative Commons Attribution License (CC BY 4.0).

\begin{abstract}
NASICON- (Na superionic conductor-) based solid-state electrolytes (SSEs) are believed to be attracting candidates for solid-state sodium batteries due to their high ionic conductivity and prospectively reliable stability. However, the poor interface compatibility and the formation of $\mathrm{Na}$ dendrites inhibit their practical application. Herein, we directly observed the propagation of $\mathrm{Na}$ dendrites through NASICON-based $\mathrm{Na}_{3.1} \mathrm{Zr}_{2} \mathrm{Si}_{2.1} \mathrm{P}_{0.9} \mathrm{O}_{12}$ SSE for the first time. Moreover, a fluorinated amorphous carbon (FAC) interfacial layer on the ceramic surface was simply developed by in situ carbonization of PVDF to improve the compatibility between Na metal and SSEs. Surprisingly, Na dendrites were effectively suppressed due to the formation of NaF in the interface when molten Na metal contacts with the FAC layer. Benefiting from the optimized interface, both the $\mathrm{Na}|| \mathrm{Na}$ symmetric cells and $\mathrm{Na}_{3} \mathrm{~V}_{2}\left(\mathrm{PO}_{4}\right)_{3} \| \mathrm{Na}$ solidstate sodium batteries deliver remarkably electrochemical stability. These results offer benign reference to the maturation of NASICON-based solid-state sodium batteries.
\end{abstract}

\section{Introduction}

Na-ion batteries (NIBs) have been investigated broadly for the potential application particularly in large-scale electrical energy storage due to the infinite sodium resources and relatively low cost [1-6]. Owning to its low electrochemical potential of $-2.71 \mathrm{~V}$ (vs. standard hydrogen electrode) and high specific capacity of $1166 \mathrm{mAhg}^{-1}$, Na metal becomes the most attractive anode for NIBs [4, 5, 7-9]. However, failures arising from the formation of $\mathrm{Na}$ dendrites and severe side reactions hinder the extensive application of NIBs adopting a $\mathrm{Na}$ metal anode coupled with organic liquid electrolytes [10]. Developing solid-state sodium batteries (SSSBs) is regarded as an effective approach to physically stabilize the
$\mathrm{Na}$ anode/solid-state electrolyte (SSE) interface, thus suppressing the $\mathrm{Na}$ dendrites and side reactions due to the high mechanical strength and wide electrochemical window of SSEs $[11,12]$. Besides, higher safety is expected due to the absence of flammable liquid electrolytes [1, 13-18].

In 1976, Goodenough et al. and Hong reported a NASICON-based SSEs, $\mathrm{Na}_{1+x} \mathrm{Zr}_{2} \mathrm{P}_{3-x} \mathrm{Si}_{x} \mathrm{O}_{12} \quad(0 \leq x \leq 3)$, which demonstrated a considerable high conductivity relying on the open 3D channels for fast $\mathrm{Na}^{+}$transport, regarded as one promising candidate of SSEs for SSSBs $[19,20]$. A modeling work by Monroe and Newman predicted that Li dendrite growth through the SSE could be mitigated if the shear modulus of the SSE was about twice that of Li metal [21]. Accordingly, the NASICON-based SSSBs are considered to not 


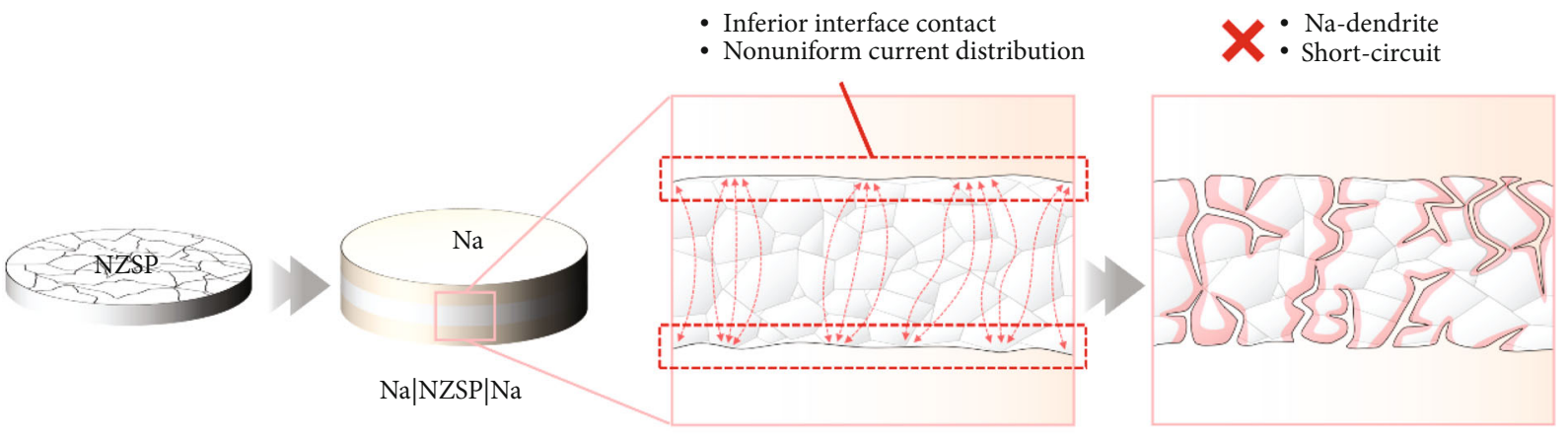

(a)

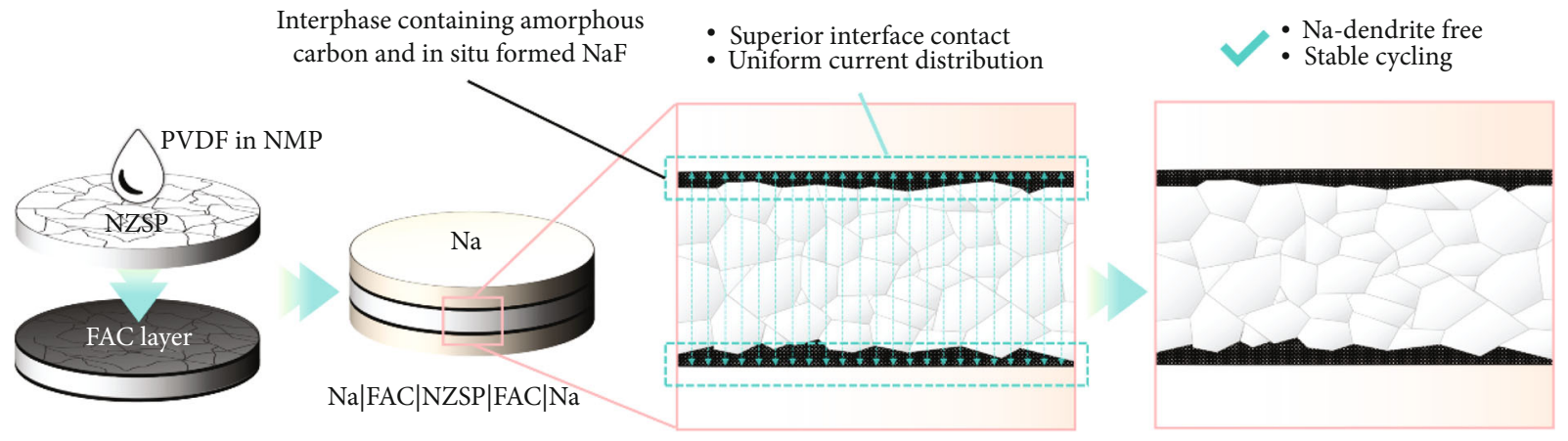

(b)

FIGURE 1: Schematic illustrations of the Na dendrite propagation in the unmodified NASICON pellet (a) and suppression in the modified NASICON pellet (b).

suffer from $\mathrm{Na}$ dendrites due to the relatively higher shear modulus of NASICON-based SSEs $(47.7 \mathrm{GPa})$ than that of $\mathrm{Na}$ metal $(3.3 \mathrm{GPa})[8,22]$. Though in principle it should work, Na dendrites still caused a short circuit in NASICONbased SSSBs [23-25]. Therefore, the shear modulus of SSEs may not be an appropriate predictor for dendrite inhibition in NASICON-based SSEs.

To suppress the $\mathrm{Na}$ dendrites, several interfacial engineering methods have been developed such as higher uniaxial compression [23], composite anode [24], 3D Na metal anode [26], chemically plating interlayer [26], in situ chemically forming interphase [27], and inserting a solid-state polymer electrolyte interlayer [27]. Despite this, the knowledge about how $\mathrm{Na}$ dendrites arise and propagate in NASICON-based SSEs is still lacking, and even the evidence of $\mathrm{Na}$ dendrites existing in NASICON-based SSEs has not been disclosed yet. Also, all of the strategies mentioned above focused on improving the wettability between NASICONbased SSEs and Na metal; however, the optimization of solid electrolyte interphase (SEI) has been ignored to a certain extent, which is also momentous for stable operation of SSSBs. On the contrary, observations towards $\mathrm{Na}$ dendrites have been carried out widely in liquid sodium batteries [10, $28-30]$. Therefore, it is urgent to directly observe $\mathrm{Na}$ dendrites in SSEs and study the formation and propagation mechanism, based on developing effective strategies to suppress the occurrence of $\mathrm{Na}$ dendrites to promote the application of NASICON-based SSEs in SSSBs.

In this study, for the first time, we directly observed the propagation of $\mathrm{Na}$ dendrites through polycrystalline
$\mathrm{Na}_{3,1} \mathrm{Zr}_{2} \mathrm{Si}_{2,1} \mathrm{P}_{0.9} \mathrm{O}_{12}$ (NZSP) SSEs. Furthermore, a fluorinated amorphous carbon (FAC) layer on the NASICONbased SSE surface was developed to improve the wettability between NZSP SSEs and Na metal anode. Meanwhile, this constructed FAC layer was reacted with molten $\mathrm{Na}$ metal by simply contacting each other to in situ form a thin SEI composed of NaF, which effectively suppresses the formation of $\mathrm{Na}$ dendrites. Benefiting from the FACregulated NZSP SSEs, extremely stable $\mathrm{Na}$ plating/stripping cycling performance at different current densities on the $\mathrm{Na}|\mathrm{FAC}| \mathrm{NASICON}|\mathrm{FAC}| \mathrm{Na}$ symmetric cells is demonstrated, and $\mathrm{Na}$ dendrites are significantly hindered. The schematic illustrations of the $\mathrm{Na}$ dendrite propagation in the unmodified NASICON pellet and suppression in the modified NASICON pellet are shown in Figure 1. Moreover, by further modification of a cathode with PEO-based solidstate polymer electrolyte (PSPE), solid-state sodium battery (SSSB) $\mathrm{Na}_{3} \mathrm{~V}_{2}\left(\mathrm{PO}_{4}\right)_{3}|\mathrm{PSPE}| \mathrm{NZSP}|\mathrm{FAC}| \mathrm{Na}$ delivered a stable cycling performance with $96.4 \%$ capacity retention after 100 cycles at $1 \mathrm{C}$ at $75^{\circ} \mathrm{C}$. Our results suggest a feasible strategy for the future development of NASICON-based SSEs in SSSBs.

\section{Materials and Methods}

2.1. Preparation of NASICON Ceramic Pellets. Nominal composition $\mathrm{Na}_{3.1} \mathrm{Zr}_{2} \mathrm{Si}_{2.1} \mathrm{P}_{0.9} \mathrm{O}_{12}$ (NZSP) SSEs were synthesized and sintered into ceramic pellets with the traditional ceramic method as reported before [31]. In a typical preparation, under a stoichiometric ratio, $\mathrm{Na}_{2} \mathrm{CO}_{3}, \mathrm{ZrO}_{2}, \mathrm{SiO}_{2}$ and 
$\mathrm{NH}_{4} \mathrm{H}_{2} \mathrm{PO}_{4}$ were ball-milled in a planetary ball mill (PM 400, Retsch) at $400 \mathrm{rpm}$ for $12 \mathrm{~h}$. As-obtained mixture was sintered at $1000^{\circ} \mathrm{C}$ for $10 \mathrm{~h}$ in air. The as-sintered product was further ball-milled under the same conditions as described above. The obtained powder was pressed into pellets with $15 \mathrm{~mm}$ in diameter and followed by a final sintering process at $1200^{\circ} \mathrm{C}$ for $20 \mathrm{~h}$ where the pellets were covered with mother powder.

2.2. Interfacial Layer for the Anode. $50 \mathrm{mg} \mathrm{mL}^{-1} \mathrm{PVDF}$ in NMP solution was spread on the polished NZSP pellets with $10 \mu \mathrm{L} \mathrm{cm}^{-2}$. After evaporating the NMP solvent, the PVDFcoated NZSP pellets were transferred into a tube furnace and sintered at $400^{\circ} \mathrm{C}$ for $2 \mathrm{~h}$ in Ar. Then, the NZSP pellets modified with fluorinated amorphous carbon (FAC) were obtained.

2.3. Material Characterization. X-ray powder diffraction (XRD) was carried out on a D8 advanced X-ray diffractometer (Bruker, Germany) using Cu K $\alpha$ radiation $(\lambda=1.5405 \AA$ ). Atomic force microscopy (AFM) was conducted on a MultiMode 8 SPM (Bruker, Germany). Electrochemical impedance spectroscopy (EIS) was performed by using the IM6e electrochemical workstation with the frequency range from $1 \mathrm{MHz}$ to $100 \mathrm{mHz}$ and an alternating current amplitude of $5 \mathrm{mV}$ (Zahner, Germany). Scanning electron microscopy (SEM) images were obtained on a Hitachi S-4800 in combination with energy-dispersive X-ray spectroscopy (EDX) mapping (Hitachi, Japan). The operando optical microanalysis is conducted on a digital microscope with AM7515 (DinoLite, China). X-ray photoelectron spectroscopy (XPS) was conducted using Thermo Scientific ESCLAB 250Xi (Thermo Fisher Scientific, America) equipped with monochromic Al $\mathrm{K} \alpha$ radiation, and all spectra were calibrated for the charging effect with the $\mathrm{C} 1 \mathrm{~s}$ peak at $284.8 \mathrm{eV}$. Transmission electron microscopy (TEM) was conducted on a JEM-2100Plus (JEOL, Japan). Thermogravimetric analysis (TGA) was conducted on a Diamond TG thermal analyzer (PerkinElmer, America).

2.4. Electrochemical Measurements. The ionic conductivity test was carried out by sputtering a gold film on both sides of the NZSP pellet as ion-blocking electrodes. To evaluate the electrochemical stability of NZSP pellets towards the $\mathrm{Na}$ metal anode, the unmodified Na|NZSP|Na symmetric cell was assembled by pressing two pieces of $\mathrm{Na}$ metal on two sides of the NZSP pellet. And the interface-modified $\mathrm{Na}|\mathrm{FAC}| \mathrm{NZSP}|\mathrm{FAC}| \mathrm{Na}$ symmetric cell was prepared by dropping molten $\mathrm{Na}$ metal onto the surface of the FACmodified NZSP pellet. All cells were assembled in a glove box under Ar. The prototype Na|NZSP|Na symmetric cell for operando optical microanalysis is shown in Supplementary Figure $\mathrm{S} 4(\mathrm{o}) . \mathrm{Na}_{3} \mathrm{~V}_{2}\left(\mathrm{PO}_{4}\right)_{3}(\mathrm{NVP})$ was prepared via a one-step ceramic process according to our previous report $[3,32]$. And the NVP cathode sheets were prepared by mixing NVP, Super P, PEO, and NaAlg in a mass ratio of $70: 15: 10: 5$ in appropriate distilled water to make a homogeneous slurry and spread on an $\mathrm{Al}$ foil, which subsequently was dried at $55^{\circ} \mathrm{C}$ under a high vacuum for
$24 \mathrm{~h}$. The average mass loading of the NVP active material is about $3 \mathrm{mg} \mathrm{cm}^{-2}$. The solid-state polymer electrolyte of PEO-NaFSI $\left(\mathrm{EO} / \mathrm{Na}^{+}=15\right.$, by molar ratio) (PSPE) was synthesized by a facile solution casting technique according to our previous report [3, 33]. To assemble solid-state batteries, the PSPE interfacial layer was put between NVP and NZSP to improve wettability, so a NVP|PSPE|NZSP|FAC|Na solid-state sodium battery was prepared. All cells were assembled in a glove box under Ar. All the galvanostatic measurements of solid-state batteries assembled in Swagelok cells were conducted on a Land BT2000 battery test system (LANHE, China).

\section{Results and Discussion}

NASICON-based $\mathrm{Na}_{3.1} \mathrm{Zr}_{2} \mathrm{Si}_{2.1} \mathrm{P}_{0.9} \mathrm{O}_{12}$ (NZSP) was synthesized and used in this study for the fabrication of SSSBs. The XRD pattern of the as-synthesized NZSP pellet matches the structure of monoclinic $\mathrm{C} 2 / \mathrm{c}$ NASICON, as confirmed by the standard diffraction peaks located at around $19.2^{\circ}$ and $27.5^{\circ}$ (Supplementary Figure S1(a)). Meanwhile, little $\mathrm{ZrO}_{2}$ impurity appears, which is common during the synthesis of NASICON-based SSEs using a ceramic method [34, 35]. The AFM image of the NZSP surface reveals a polycrystalline structure and dense morphology (Supplementary Figure S1(b)). The resistance of NZSP (thickness of $1.2 \mathrm{~mm}$ and diameter of $11.2 \mathrm{~mm}$ ) was measured by EIS at temperatures from $15^{\circ} \mathrm{C}$ to $75^{\circ} \mathrm{C}$. The conductivities were calculated and fitted well with the Arrhenius equation to demonstrate a good linear relationship with an activation energy of $0.33 \mathrm{eV}$ (Supplementary Figure S1(c)). The Nyquist plots at $25^{\circ} \mathrm{C}$ and $75^{\circ} \mathrm{C}$ are shown in Supplementary Figure S1(d), and high conductivities of $1.04 \mathrm{mS} \mathrm{cm}^{-1}$ at $25^{\circ} \mathrm{C}$ and $5.84 \mathrm{mS} \mathrm{cm}^{-1}$ at $75^{\circ} \mathrm{C}$ are achieved.

For SSSBs, besides the high ionic conductivity of SSEs, the intimate contact with electrodes to enable a stable and compatible electrode|SSE interface for sufficient ion conduction is the key to improve the electrochemical performances of SSSBs. When the untreated NZSP pellets are employed, the pressed $\mathrm{Na}$ metal may not wet the ceramic, forming an inhomogeneous and incompatible interface with many voids and roughness as shown in Figure 1(a) $[24,26]$. As a result, the $\mathrm{Na}^{+}$preferentially plates on these voids, roughness, and grain boundaries, where the electric field is locally enhanced, leading to the nucleation and propagation of $\mathrm{Na}$ dendrites during $\mathrm{Na}$ plating/stripping cycling [27]. Furthermore, $\mathrm{Na}$ dendrites can penetrate the interconnected grain boundaries of ceramic and cause a short circuit. To quantitatively evaluate the effect of the untreated $\mathrm{NZSP} \mid \mathrm{Na}$ interface, the $\mathrm{Na}|\mathrm{NZSP}| \mathrm{Na}$ symmetric cells were assembled and measured by EIS to obtain the interfacial resistance. The Nyquist plot at $75^{\circ} \mathrm{C}$ (Supplementary Figure S2) presents a semicircle at medium frequencies associated with the interface response [36]. The interfacial resistance of about $100 \Omega$ is reached, which possibly results from the existing voids and roughness in the interface. After that, the symmetric cell was cycled by periodically charging and discharging, respectively, for $1 \mathrm{~h}$ to evaluate the interfacial stability and 


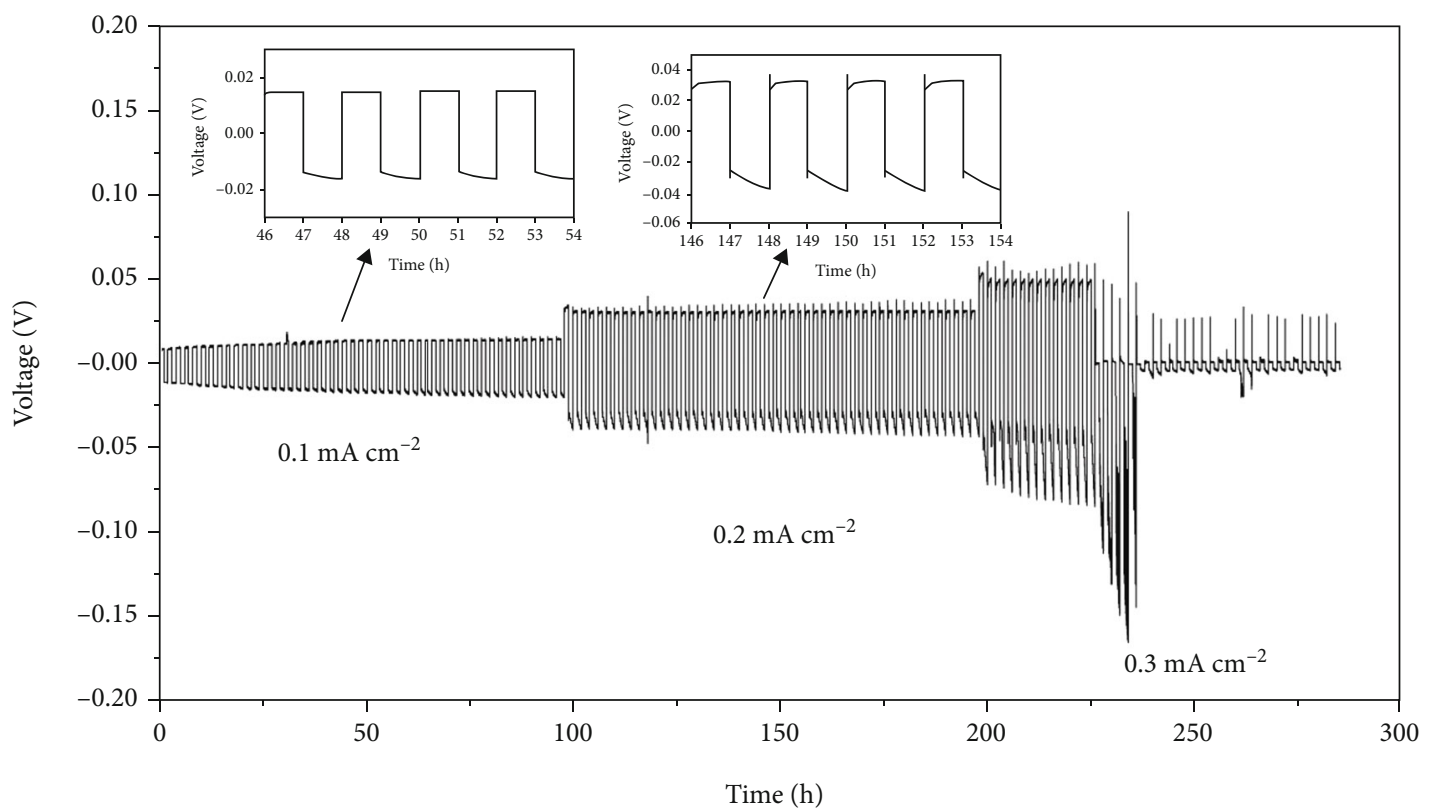

(a)

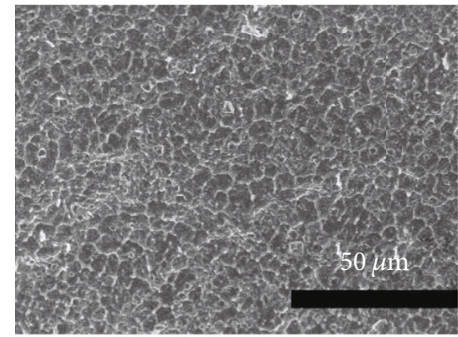

(b)

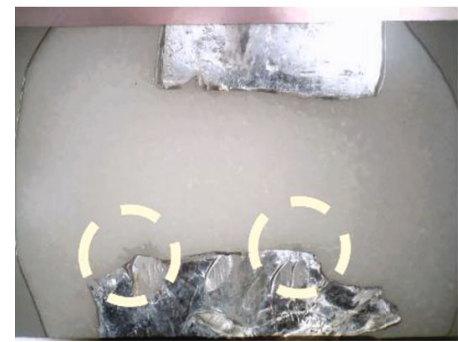

(e)

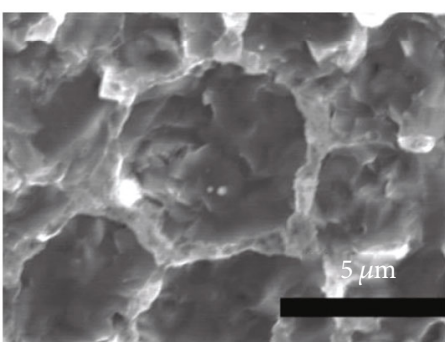

(c)

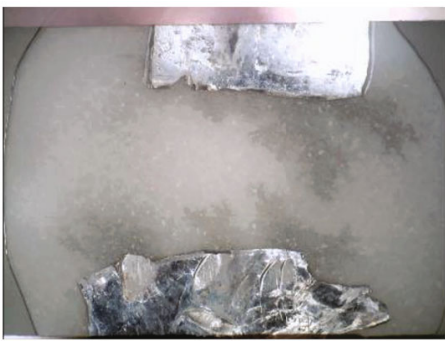

(f)

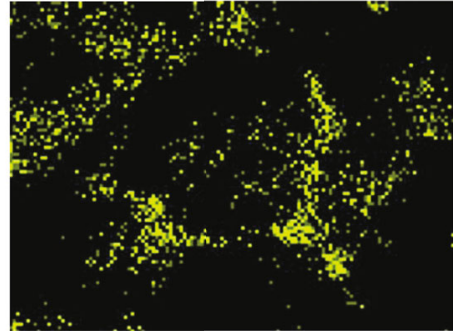

(d)

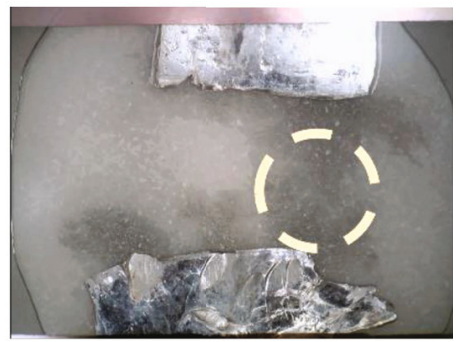

(g)

FIgure 2: (a) Galvanostatic cycling of the Na|NASICON|Na symmetric cell at different current densities at $75^{\circ} \mathrm{C}$. (b, c) Web-structured dendrite and (d) corresponding EDX mapping. Operando optical observations of Na dendrite propagation in NASICON under (e) $10 \mu \mathrm{A}$, (f) $70 \mu \mathrm{A}$, and (g) $130 \mu \mathrm{A}$ for a 2-minute charging process and 2-minute discharging process within the identical cell at prolonged cycling times.

detect whether $\mathrm{Na}$ dendrites are formed during this process. As shown in Figure 2(a), ohmic behavior is observed at low current densities less than $0.3 \mathrm{~mA} \mathrm{~cm}^{-2}$. It is worth noting that the profiles of charging and discharging processes are getting noisier with increasing current density and capacity; besides, the overpotential of the discharging process is less stable than that of the charging process because of asymmetrical volume changes of the cathode and anode [37]. At $0.3 \mathrm{~mA} \mathrm{~cm}^{-2}$, the symmetric cell undergoes an abrupt voltage drop after polarization for a short period, which may be induced by $\mathrm{Na}$ dendrite formation, resulting in the occurrence of the short circuit. $\mathrm{Na}$ metal is subjected to $100 \%$ volumetric expansion/contraction upon repeated cycling due to its hostless nature [4]. Usually, the plating/stripping of $\mathrm{Na}$ metal happens on the interface between SSEs and $\mathrm{Na}$ metal, preferentially at the local area but not the whole interface; as a consequence, locally intensified current density near the voids, roughness, grain boundaries, etc. exacerbates the $\mathrm{Na}$ dendrite formation during heterogeneous plating/stripping. Besides, it is 
reported that NASICON-based SSEs are not stable towards $\mathrm{Na}$ metal, and some side reactions have been observed even at room temperature, which partially expedites $\mathrm{Na}$ dendrite formation $[23,38]$.

To examine the reasons for the short circuit, the symmetric cell was disassembled and a clear dark line is observed on the surface of cycled NZSP pellets as shown in the optical photograph of Supplementary Figure S3. Then, we broke the pellet along the dark line for the SEM analysis. The characteristic region of the fracture was detected with a web-like morphology (Figures 2(b) and 2(c)). To further analyze the composition of the detected regions, EDX mapping was conducted and composed of $\mathrm{Na}$ element (Figure 2(d)). It is interesting to note that the web-like morphology is very similar to that of the intergranular $\mathrm{Li}$ dendrites observed in garnet SSEs as reported by Cheng et al. [39]. Therefore, $\mathrm{Na}$ dendrites are confirmed according to the above analysis, which is visually observed in the NZSP electrolyte for the first time to the best of our knowledge. According to previous investigations, we believe that the inhomogeneous current distribution induced by the nonuniform interfacial connection between $\mathrm{Na}$ metal and SSEs, the infinite volume change of $\mathrm{Na}$ metal, and the unreliable SEI are the key to cause $\mathrm{Na}$ dendrite formation, thus inducing the short circuit. Besides, due to the polycrystal essence and the different $\mathrm{Na}^{+}$conductivities in grain and grain boundary of NASICON, the $\mathrm{Na}$ metal would propagate along the NASICON grain boundaries to form a web [39]. Furthermore, for observing the $\mathrm{Na}$ dendrite propagation in the NASICON pellet more intuitively, operando optical microanalysis was conducted. Figures $2(\mathrm{e})-2(\mathrm{~g})$ show optical images of $\mathrm{Na}$ dendrites after 2-minute charging and 2-minute discharging processes at $10 \mu \mathrm{A}, 70 \mu \mathrm{A}$, and $130 \mu \mathrm{A}$, respectively. And more images under different current are provided in Supplementary Figure S4; meanwhile, the movie of the operando optical microanalysis is provided in Supplementary Movie S1. From which, it can be seen that $\mathrm{Na}$ dendrites preferentially germinate on the tips where the electric field is locally enhanced as circled in Figure 2(e). Besides, with increasing current density, the $\mathrm{Na}$ dendrites gradually propagate towards the counter electrode and get short circuit at $130 \mu \mathrm{A}$ as circled in Figure 2(g). More seriously, once the $\mathrm{Na}$ dendrites are formed, they do not disappear but continue to grow on the original basis until getting short circuit. Hence, it is urgent to realize the dendrite-free NASICON-based SSEs by interfacial modification.

Carbonaceous materials, regarded as excellent interface layers and/or host frames for metal electrodes due to their high electronic conductivity and flexible nature, have been widely employed as modifying material in liquid- and solidstate batteries [40-44]. Besides, it is believed that the presence of $\mathrm{NaF}$ in SEI is effective by its stabilizing effect on the $\mathrm{Na}$ metal surface for enabling dendrite-free, uniform, and dense Na plating $[8,45,46]$. Therefore, if a carbon interface layer can be constructed between the $\mathrm{Na}$ metal anode and NZSP SSE with an in situ-formed SEI containing NaF, SSSBs are expected to deliver a splendid performance. Inspired by this idea, we chose PVDF with a high content of both C and $\mathrm{F}$ elements to build the interface layer. PVDF can be carbonized at low temperatures in Ar as confirmed by TGA as presented in Supplementary Figure S5(a). The in situ carbonization of PVDF on the surface of the NZSP pellet was realized as shown in Figure 3(a), where the inset map confirms the amorphous nature of PVDF-derived carbon. The lateral digital photo in Figure 3(b) demonstrates the excellent wetting of FAC-modified NZSP towards Na metal, and the top view is shown in Supplementary Figure S5(b). Furthermore, the SEM image in Figure 3(c) confirms the close connection between $\mathrm{Na}$ metal and FAC-modified NZSP pellets, where the sodiated FAC layer modifies the uneven surface of the NZSP pellet effectively. EDX mapping further reveals that the FAC layer can effectively improve the wetting property of NZSP SSEs with Na metal as shown in Figures 3(d)-3(f).

The XPS spectra shown in Figures 3(g) and 3(h) are the C1s, F1s, and Na1s spectra of the FAC layer before (Figure 3(g)) and after (Figure 3(h)) contacting with molten $\mathrm{Na}$. The fitted XPS spectra of C1s and F1s in the original FAC layer indicate that the $\mathrm{F}$ element exists in C-F bonds as shown in Figure 3(g), which further suggests the fluorinated amorphous carbon nature of the PVDF-derived interfacial layer. As expected, the Na element from NZSP is not detected due to the fact that the thickness of FAC exceeds the detecting depth of XPS. After reaction with molten $\mathrm{Na}$ metal, C1s and F1s in FAC remarkably changed as presented in Figure 3(h). The C1s peaks of F-C-C* and F-C $*$ disappear, and the F1s peak shifts towards lower binding energy, suggesting the breaking of C-F bonds and generation of $\mathrm{Na}-\mathrm{F}$ bonds. Besides, the fitted XPS spectrum of Na1s further confirms the reaction between molten $\mathrm{Na}$ metal and the FAC layer with the formation of $\mathrm{NaF}$.

According to the above results, the interphase composed of the carbonaceous material and $\mathrm{NaF}$ inorganic salt was successively in situ formed as our original design. To verify the effect of FAC modification on the improvement of the Na|NZSP interface, EIS was used to quantify the change of the interfacial resistance. One half of the decrease is observed in Supplementary Figure S2(b) compared with original interfacial resistance as shown in Supplementary Figure S2(a). The decreased resistance can be attributed to the better connection between $\mathrm{Na}$ metal and NZSP pellets due to the modification of the FAC layer, which alleviates surface voids and roughness and provides superior wetting behavior. Galvanostatic cycling experiments are carried out to evaluate the capability of suppressing $\mathrm{Na}$ dendrites and the diffusion of $\mathrm{Na}^{+}$across the interface as shown in Figure 4 . At $0.1 \mathrm{~mA} \mathrm{~cm}^{-2}$ current density, the Na|FAC $|\mathrm{NZSP}| \mathrm{FAC} \mid \mathrm{Na}$ cell stabilizes at around $6 \mathrm{mV}$, and stable cycling below $0.6 \mathrm{~mA} \mathrm{~cm}^{-2}$ is reached, well according to the ohmic behavior. It is worth noting that the polarization voltages of charging and discharging processes get more stable than those of the symmetric cell based on the untreated NZSP. The asymmetrical effect of volume changes at the cathode and anode has been suppressed benefitting from the flexible nature of the FAC layer and the homogeneous $\mathrm{Na}^{+}$flux across the interface. More importantly, there is no abrupt 


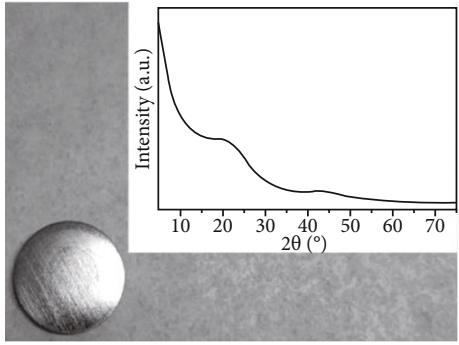

(a)

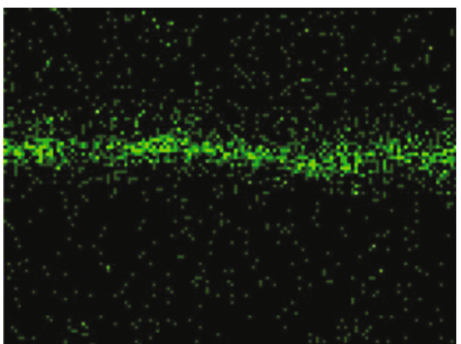

(d)

$$
\mathrm{C} 1 \mathrm{~s}
$$

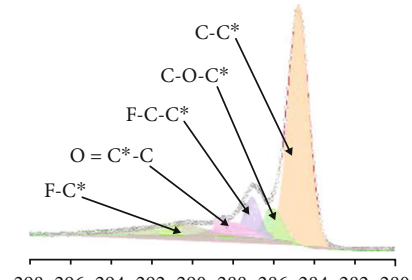

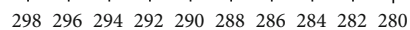

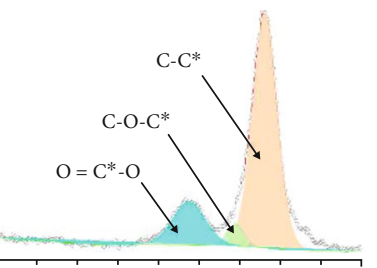

298296294292290288286284282280

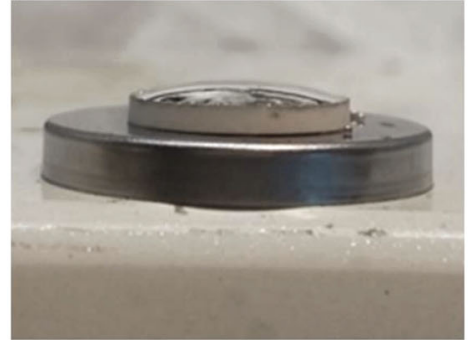

(b)

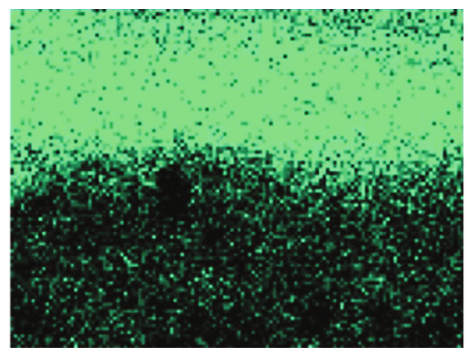

(e)

F1s

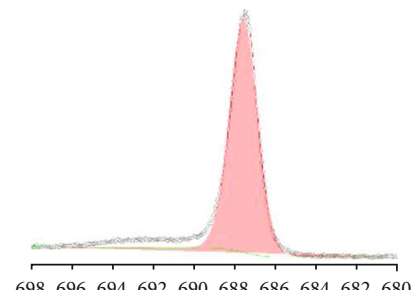

$\begin{array}{llllllllll}698 & 696 & 694 & 692 & 690 & 688 & 686 & 684 & 682 & 680\end{array}$

Binding energy $(\mathrm{eV})$

(g)

F1s

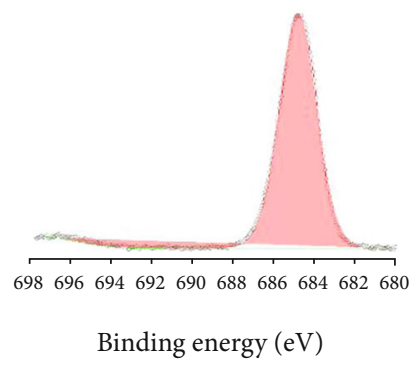

(h)

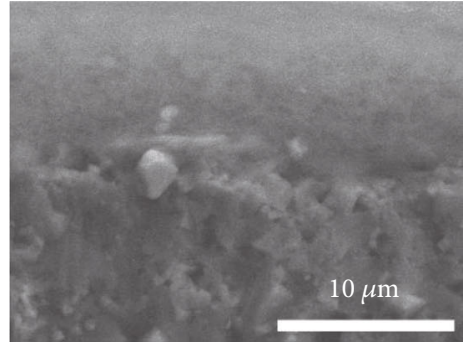

(c)

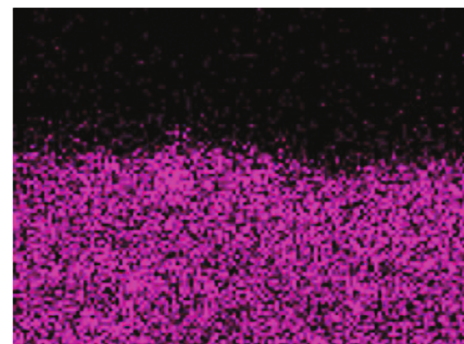

(f)

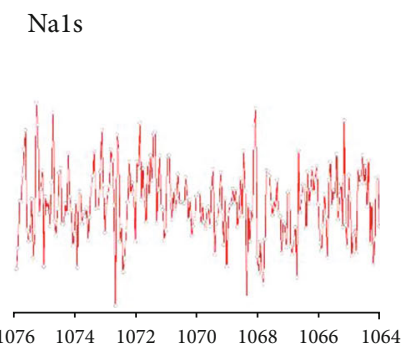

$\mathrm{Na} 1 \mathrm{~s}$

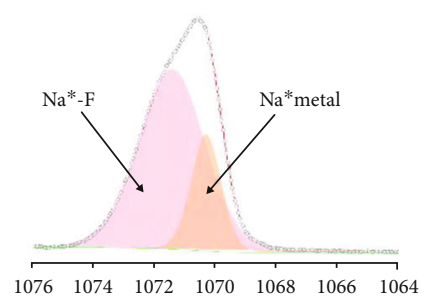

FIgURE 3: FAC-treated NZSP. (a) Digital photo of the PVDF-derived layer on the surface of NZSP. Inset is the XRD profile of PVDF-derived carbon. (b) Optical photograph of melted Na metal on top of the NZSP pellet. (c) SEM image of the FAC-treated NZSP pellet. EDX mapping of (d)-(f) revealing the distribution of F, Na, and P elements near the interface between Na metal and NZSP pellet. C1s, F1s, and Na1s XPS spectra from (g) untreated and (h) FAC-treated NZSP pellets.

drop in the overpotential when polarization is at $0.6 \mathrm{~mA} \mathrm{~cm}^{-}$ ${ }^{2}$; inversely, an abrupt open circuit is observed for a period. This phenomenon confirms the excellent capability of the FAC layer to suppress $\mathrm{Na}$ dendrites. All the results suggest that the FAC layer realizes the excellent interfacial contact, facilitates the $\mathrm{Na}^{+}$diffusion across the interface, improves the interfacial stability, and especially possesses a superior capability of suppressing $\mathrm{Na}$ dendrites.
To evaluate the electrochemical performance of FACmodified NZSP, SSSBs were assembled with NVP as a cathode, $\mathrm{Na}$ metal as an anode, and FAC-modified NZSP as the electrolyte. The synthesized NVP cathode delivers a pure rhombohedral R-3c structure as confirmed by XRD (Supplementary Figure S6(a)). The SEM image (Supplementary Figure S6(b)) confirms the uniformly distributed nanoparticles. The HRTEM image (Supplementary 


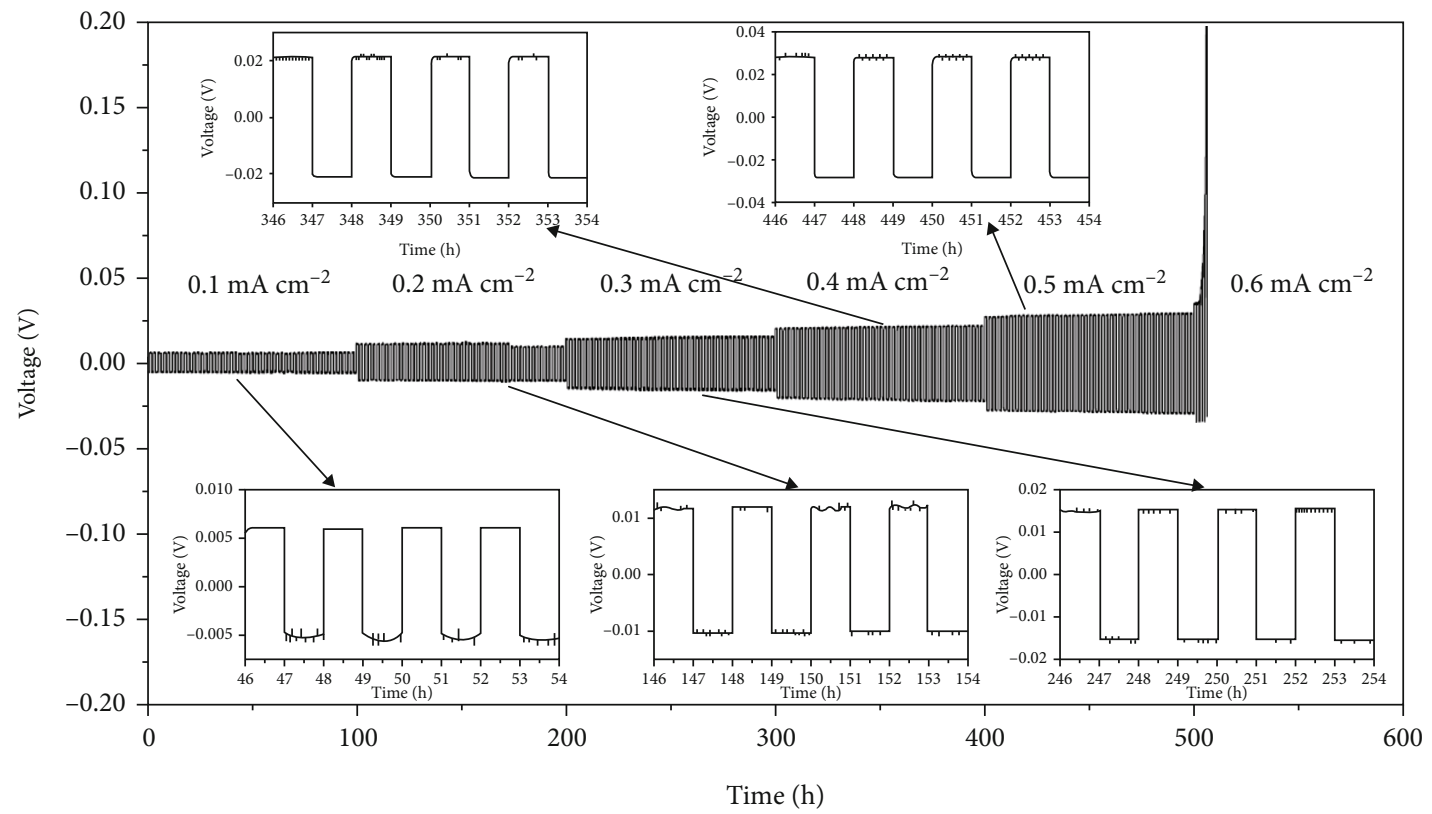

FIgURE 4: Galvanostatic cycling of the Na|NASICON|Na symmetric cell at different current densities at $75^{\circ} \mathrm{C}$.

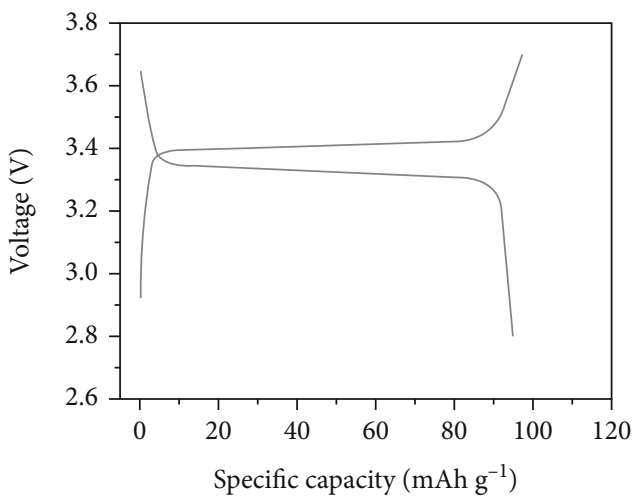

(a)

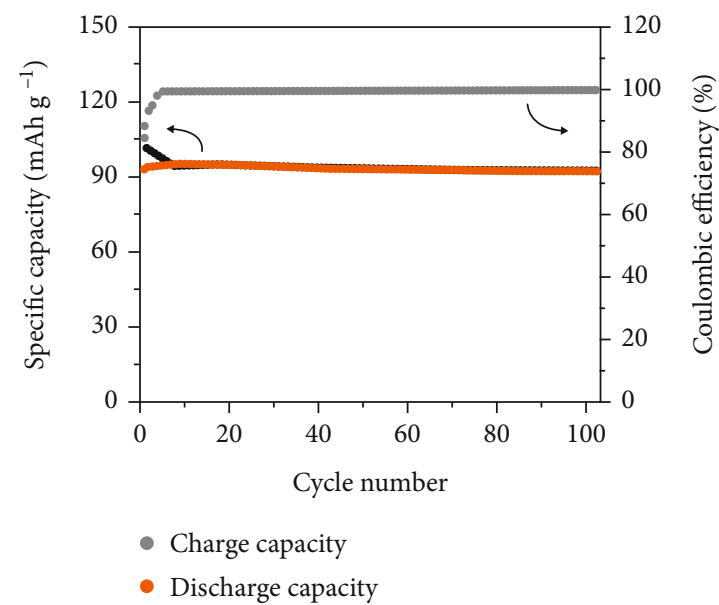

(b)

FIgURE 5: NVP|PSPE|NZSP|FAC|Na ASSSB at $75^{\circ} \mathrm{C}$ : (a) the galvanostatic charge and discharge profile at $1 \mathrm{C}$; (b) cycling performance at $1 \mathrm{C}$ $\left(0.3 \mathrm{~mA} \mathrm{~cm}^{-2}\right)$.

Figure S6(c)) shows a homogenous 4 nm carbon coating layer on the NVP particle and the corresponding lattice fingers of (104) and (102) planes of NVP. Supplementary Figure S6(d) shows the SEAD image corresponding to the area shown in Supplementary Figure S6(c), which further confirms the rhombohedral structure of the as-synthesized NVP. Besides, to wet the interface between NVP and NZSP, PEO-NaFSI PSPE was prepared and inserted. And the good compatibility between NVP and PSPE has been illustrated elsewhere $[33,47]$. The Arrhenius plot of $\mathrm{Na}^{+}$conductivity of PSPE is shown in Supplementary Figure S7(a), and high ionic conductivity of $0.432 \mathrm{mS} \mathrm{cm}^{-1}$ at $75^{\circ} \mathrm{C}$ is reached. Moreover, as presented in Supplementary Figure S7(b), the high thermal decomposition temperature of around $240^{\circ} \mathrm{C}$ ensures the high safety of ASSSBs. A high initial discharge capacity of $95.2 \mathrm{mAhg}^{-1}$ and $97.84 \%$ Coulombic efficiency at $1 \mathrm{C}$ were achieved for NVP|PSPE|NZSP|FAC|Na ASSSB as shown in Figure 5(a), before which a low current density of $0.1 \mathrm{C}$ was applied to aim at electrochemically forming interphases for both the cathode and anode. Supplementary Figure S8 shows the first 3 cycles of charging and discharging profiles at $0.1 \mathrm{C}$. After 100 cycles at $1 \mathrm{C}$, the ASSSB demonstrates $96.4 \%$ capacity retention with $91.8 \mathrm{mAh} \mathrm{g}^{-1}$ and nearly $100 \%$ Coulombic efficiency as shown in Figure 5(b). The superior cycling stability could be attributed to the excellent wettability of PSPE towards the NZSP and NVP cathode, and more importantly, that the FAC interfacial layer provides the splendid interface reliability between NZSP and $\mathrm{Na}$ metal anodes, ensuring interfacial contact and suppressing $\mathrm{Na}$ dendrites. Besides, 
the $\mathrm{NaF}$ in the interfacial layer also contributes to enabling uniform and dense Na plating.

\section{Conclusion}

In summary, we presented direct evidence for the first time that $\mathrm{Na}$ dendrites can propagate through the hard NASICON ceramic, which is crucial for understanding the mechanism of occurrence and propagation of $\mathrm{Na}$ dendrites in NASICON-based solid-state electrolytes. Furthermore, we developed a FAC layer on the NASICON ceramic pellet surface by in situ carbonization of PVDF to improve the wettability between $\mathrm{Na}$ metal and NASICON, where $\mathrm{NaF}$ was in situ formed in the SEI to enhance the interfacial stability. Benefiting from the optimized interphase, the $\mathrm{Na} \| \mathrm{Na}$ symmetric cell shows much better cycling stability, significantly inhibiting the sodium dendrites. Combined with the PSPE cathode interlayer, the fabricated NVP|PSPE|NZSP|FAC|Na SSSBs delivered excellent electrochemical stability, demonstrating $96.4 \%$ capacity retention at $1 \mathrm{C}$ after 100 cycles. The results in this study are expected to provide a feasible strategy for the research and application of NASICON-based SSEs in SSSBs.

\section{Data Availability}

The authors declare that the main data supporting the findings in this study are available within the article and its supplementary information. Additional data are available from the corresponding authors upon reasonable request.

\section{Conflicts of Interest}

The authors declare no competing interests.

\section{Authors' Contributions}

Y.X.L., Y-S.H., X.G.H., H.L., and L.Q.C. proposed and designed the project. Q.Q.Z. synthesized the electrolyte and electrode materials and performed electrochemical measurements and carried out characterizations. W.C.G. performed the operando optical microanalysis. Y.J.S. and L.L.L. performed the XPS analysis. J.Z.L. carried out the AFM characterization. X.H.R. drew the schematic diagram of Figure 1. Q.Q.Z, Y.X.L., Y-S.H., and X.G.H. wrote the manuscript. All authors discussed the results and commented on the manuscript.

\section{Acknowledgments}

This work was supported by the National Key Technologies R\&D Program, China (2016YFB0901500); the Opening Project of Key Laboratory of Optoelectronic Chemical Materials and Devices, Ministry of Education, Jianghan University (JDGD-201703); the National Natural Science Foundation of China (51725206); the Strategic Priority Research Program of the Chinese Academy of Sciences (XDA21070500); and Youth Innovation Promotion Association, Chinese Academy of Sciences (2020006).

\section{Supplementary Materials}

Supplementary 1. Figure $\mathrm{S} 1: \mathrm{Na}_{3.1} \mathrm{Zr}_{2} \mathrm{Si}_{2.1} \mathrm{P}_{0.9} \mathrm{O}_{12}$. (a) $\mathrm{XRD}$ patterns (C2/c, COD: 1529608; R-3c, COD: 1529809). (b) AFM morphology of the surface. (c) Arrhenius plot of total conductivity vs. reciprocal temperature. (d) Nyquist plot at $25^{\circ} \mathrm{C}$ and $75^{\circ} \mathrm{C}$. Figure S2: Na||Na symmetric cell. (a) EIS profile for the $\mathrm{Na}|\mathrm{NZSP}| \mathrm{Na}$ symmetric cell at $75^{\circ} \mathrm{C}$. (b) EIS profile for the Na|FAC|NZSP|FAC|Na symmetric cell at $75^{\circ} \mathrm{C}$. Figure S3: optical photograph of the short-circuited NZSP. Figure S4: operando optical observations of $\mathrm{Na}$ dendrite propagation in NASICON under different current for a 2-minute charging process and 2-minute discharging process. (a) Origin. (b) $10 \mu \mathrm{A}$. (c) $20 \mu \mathrm{A}$. (c) $30 \mu \mathrm{A}$. (e) $40 \mu \mathrm{A}$. (f) $50 \mu \mathrm{A}$. (g) $60 \mu \mathrm{A}$. (h) $70 \mu \mathrm{A}$. (i) $80 \mu \mathrm{A}$. (j) $90 \mu \mathrm{A}$. (k) $100 \mu \mathrm{A}$. (l) $110 \mu \mathrm{A}$. (m) $120 \mu \mathrm{A}$. (n) $130 \mu \mathrm{A}$. (o) Optical photograph of the $\mathrm{Na}|| \mathrm{Na}$ symmetric cell after cycling. Figure S5: (a) TGA trace of PVDF from $50^{\circ} \mathrm{C}$ to $800^{\circ} \mathrm{C}$. (b) Digital photo of molten Na metal on top of the NZSP surface. Figure S6: $\mathrm{Na}_{3} \mathrm{~V}_{2}\left(\mathrm{PO}_{4}\right)_{3}$. (a) XRD pattern. (b) SEM image. (c) HRTEM image. (d) Corresponding SEAD pattern. Figure S7: PEO15-NaFSI. (a) Ionic conductivity at different temperatures. (b) TGA trace from $50^{\circ} \mathrm{C}$ to $650^{\circ} \mathrm{C}$. Figure S8: the galvanostatic charge and discharge profile for the first 3 cycles of NVP|PSPE|NZSP|FAC|Na ASSSB at $0.1 \mathrm{C}$ and $75^{\circ} \mathrm{C}$. The low Coulombic efficiencies in first 3 cycles $(84.37 \%, 93.13$, and $94.8 \%$ ) contributed to the formations of SEI at the anode and CEI at the cathode.

Supplementary 2. Movie S1: the movie of the operando optical microanalysis.

\section{References}

[1] J. Yang, G. Liu, M. Avdeev et al., "Ultrastable all-solid-state sodium rechargeable batteries," ACS Energy Letters, vol. 5, no. 9, pp. 2835-2841, 2020.

[2] E. Goikolea, V. Palomares, S. Wang et al., "Na-ion batteries-approaching old and new challenges," Advanced Energy Materials, vol. 10, no. 44, article 2002055, 2020.

[3] Q. Zhang, Y. Lu, H. Yu et al., "PEO-NaPF 6 blended polymer electrolyte for solid state sodium battery," Journal of the Electrochemical Society, vol. 167, no. 7, article 070523, 2020.

[4] X. Zheng, C. Bommier, W. Luo, L. Jiang, Y. Hao, and Y. Huang, "Sodium metal anodes for room-temperature sodium-ion batteries: applications, challenges and solutions," Energy Storage Materials, vol. 16, pp. 6-23, 2019.

[5] Y. Wang, Y. Wang, Y.-X. Wang et al., "Developments and perspectives on emerging high-energy-density sodium-metal batteries," Chem, vol. 5, no. 10, pp. 2547-2570, 2019.

[6] Y.-S. Hu and Y. Lu, "2019 Nobel Prize for the Li-ion batteries and new opportunities and challenges in Na-ion batteries," ACS Energy Letters, vol. 4, no. 11, pp. 2689-2690, 2019.

[7] B. Sun, P. Xiong, U. Maitra et al., "Design strategies to enable the efficient use of sodium metal anodes in high-energy batteries," Advanced Materials, vol. 32, no. 18, article 1903891, 2020.

[8] B. Lee, E. Paek, D. Mitlin, and S. W. Lee, "Sodium metal anodes: emerging solutions to dendrite growth," Chemical Reviews, vol. 119, no. 8, pp. 5416-5460, 2019. 
[9] L. Fan and X. Li, "Recent advances in effective protection of sodium metal anode," Nano Energy, vol. 53, pp. 630-642, 2018.

[10] R. Rodriguez, K. E. Loeffler, S. S. Nathan et al., "In situ optical imaging of sodium electrodeposition: effects of fluoroethylene carbonate," ACS Energy Letters, vol. 2, no. 9, pp. 2051-2057, 2017.

[11] Y. Yao, Z. Wei, H. Wang et al., "Toward high energy density all solid-state sodium batteries with excellent flexibility," Advanced Energy Materials, vol. 10, no. 12, article 1903698, 2020.

[12] F. Wu, K. Zhang, Y. Liu et al., "Polymer electrolytes and interfaces toward solid-state batteries: recent advances and prospects," Energy Storage Materials, vol. 33, pp. 26-54, 2020.

[13] Q. Zhao, S. Stalin, C.-Z. Zhao, and L. A. Archer, "Designing solid-state electrolytes for safe, energy-dense batteries," Nature Reviews Materials, vol. 5, no. 3, pp. 229-252, 2020.

[14] L. Qiao, X. Judez, T. Rojo, M. Armand, and H. Zhang, "Review-polymer electrolytes for sodium batteries," Journal of the Electrochemical Society, vol. 167, no. 7, article 070534, 2020.

[15] C. Zhou, S. Bag, and V. Thangadurai, "Engineering materials for progressive all-solid-state Na batteries," ACS Energy Letters, vol. 3, no. 9, pp. 2181-2198, 2018.

[16] G. Chen, Y. Bai, Y. Gao et al., "Inhibition of crystallization of poly(ethylene oxide) by ionic liquid: insight into plasticizing mechanism and application for solid-state sodium ion batteries," ACS Applied Materials \& Interfaces, vol. 11, no. 46, pp. 43252-43260, 2019.

[17] G. Chen, L. Ye, K. Zhang et al., "Hyperbranched polyether boosting ionic conductivity of polymer electrolytes for allsolid-state sodium ion batteries," Chemical Engineering Journal, vol. 394, article 124885, 2020.

[18] G. Chen, K. Zhang, Y. Liu et al., "Flame-retardant gel polymer electrolyte and interface for quasi-solid-state sodium ion batteries," Chemical Engineering Journal, vol. 401, article 126065, 2020.

[19] J. B. Goodenough, H. Y. P. Hong, and J. A. Kafalas, "Fast $\mathrm{Na}^{+}-$ ion transport in skeleton structures," Materials Research Bulletin, vol. 11, no. 2, pp. 203-220, 1976.

[20] H. Y. P. Hong, "Crystal structures and crystal chemistry in the system $\mathrm{Na}_{1+\mathrm{x}} \mathrm{Zr}_{2} \mathrm{Si}_{\mathrm{x}} \mathrm{P}_{3-\mathrm{x}} \mathrm{O}_{12}$," Materials Research Bulletin, vol. 11, no. 2, pp. 173-182, 1976.

[21] C. Monroe and J. Newman, "The impact of elastic deformation on deposition kinetics at lithium/polymer interfaces," Journal of the Electrochemical Society, vol. 152, no. 2, article A396, 2005.

[22] Z. Deng, Z. Wang, I.-H. Chu, J. Luo, and S. P. Ong, "Elastic properties of alkali superionic conductor electrolytes from first principles calculations," Journal of the Electrochemical Society, vol. 163, no. 2, pp. A67-A74, 2015.

[23] Y. Uchida, G. Hasegawa, K. Shima et al., "Insights into sodium ion transfer at the $\mathrm{Na} / \mathrm{NASICON}$ interface improved by uniaxial compression," ACS Applied Energy Materials, vol. 2, no. 4, pp. 2913-2920, 2019.

[24] H. Fu, Q. Yin, Y. Huang et al., "Reducing interfacial resistance by $\mathrm{Na}-\mathrm{SiO}_{2}$ composite anode for NASICON-based solid-state sodium battery," ACS Materials Letters, vol. 2, no. 2, pp. 127-132, 2020.

[25] A. V. Virkar and L. Viswanathan, "Sodium penetration in rapid ion conductors," Journal of the American Ceramic Society, vol. 62, no. 9-10, pp. 528-529, 1979.
[26] Y. Lu, J. A. Alonso, Q. Yi, L. Lu, Z. L. Wang, and C. Sun, “A high-performance monolithic solid-state sodium battery with $\mathrm{Ca}^{2+}$ doped $\mathrm{Na}_{3} \mathrm{Zr}_{2} \mathrm{Si}_{2} \mathrm{PO}_{12}$ electrolyte," Advanced Energy Materials, vol. 9, no. 28, article 1901205, 2019.

[27] W. Zhou, Y. Li, S. Xin, and J. B. Goodenough, "Rechargeable sodium all-solid-state battery," ACS Central Science, vol. 3, no. 1, pp. 52-57, 2017.

[28] S. Wei, S. Choudhury, J. Xu, P. Nath, Z. Tu, and L. A. Archer, "Highly stable sodium batteries enabled by functional ionic polymer membranes," Advanced Materials, vol. 29, no. 12, article 1605512, 2017.

[29] L. Medenbach, C. L. Bender, R. Haas et al., "Origins of dendrite formation in sodium-oxygen batteries and possible countermeasures," Energy Technology, vol. 5, no. 12, pp. 2265-2274, 2017.

[30] S. Choudhury, S. Wei, Y. Ozhabes et al., "Designing solidliquid interphases for sodium batteries," Nature Communications, vol. 8, no. 1, p. 898, 2017.

[31] Y. Shao, G. Zhong, Y. Lu et al., "A novel NASICON-based glass-ceramic composite electrolyte with enhanced Na-ion conductivity," Energy Storage Materials, vol. 23, pp. 514-521, 2019.

[32] Z. Jian, W. Han, X. Lu et al., "Superior electrochemical performance and storage mechanism of $\mathrm{Na}_{3} \mathrm{~V}_{2}\left(\mathrm{PO}_{4}\right)_{3}$ cathode for room-temperature sodium-ion batteries," Advanced Energy Materials, vol. 3, no. 2, pp. 156-160, 2013.

[33] X. Qi, Q. Ma, L. Liu et al., "Sodium bis(fluorosulfonyl)imide/poly(ethylene oxide) polymer electrolytes for sodium-ion batteries," ChemElectroChem, vol. 3, no. 11, pp. 1741-1745, 2016.

[34] M. Samiee, B. Radhakrishnan, Z. Rice et al., "Divalent-doped $\mathrm{Na}_{3} \mathrm{Zr}_{2} \mathrm{Si}_{2} \mathrm{PO}_{12}$ natrium superionic conductor: improving the ionic conductivity via simultaneously optimizing the phase and chemistry of the primary and secondary phases," Journal of Power Sources, vol. 347, pp. 229-237, 2017.

[35] U. Von Alpen, M. F. Bell, and H. H. Höfer, "Compositional dependence of the electrochemical and structural parameters in the NASICON system $\left(\mathrm{Na}_{1+\mathrm{x}} \mathrm{Si}_{\mathrm{x}} \mathrm{Zr}_{2} \mathrm{P}_{3-\mathrm{x}} \mathrm{O}_{12}\right)$," Solid State Ionics, vol. 3-4, pp. 215-218, 1981.

[36] J. T. S. Irvine, D. C. Sinclair, and A. R. West, "Electroceramics: characterization by impedance spectroscopy," Advanced Materials, vol. 2, no. 3, pp. 132-138, 1990.

[37] W. Manalastas, J. Rikarte, R. J. Chater et al., "Mechanical failure of garnet electrolytes during Li electrodeposition observed by in-operando microscopy," Journal of Power Sources, vol. 412, pp. 287-293, 2019.

[38] S. Wang, H. Xu, W. Li, A. Dolocan, and A. Manthiram, "Interfacial chemistry in solid-state batteries: formation of interphase and its consequences," Journal of the American Chemical Society, vol. 140, no. 1, pp. 250-257, 2018.

[39] E. J. Cheng, A. Sharafi, and J. Sakamoto, "Intergranular Li metal propagation through polycrystalline $\mathrm{Li}_{6.25} \mathrm{Al}_{0.25}$ $\mathrm{La}_{3} \mathrm{Zr}_{2} \mathrm{O}_{12}$ ceramic electrolyte," Electrochimica Acta, vol. 223, pp. 85-91, 2017.

[40] S.-S. Chi, X.-G. Qi, Y.-S. Hu, and L.-Z. Fan, “3D flexible carbon felt host for highly stable sodium metal anodes," Advanced Energy Materials, vol. 8, no. 15, article 1702764, 2018.

[41] W.-S. Xiong, Y. Jiang, Y. Xia et al., "A robust 3D host for sodium metal anodes with excellent machinability and cycling stability," Chemical Communications, vol. 54, no. 68, pp. 9406-9409, 2018. 
[42] W. Luo, Y. Zhang, S. Xu et al., "Encapsulation of metallic Na in an electrically conductive host with porous channels as a highly stable $\mathrm{Na}$ metal anode," Nano Letters, vol. 17, no. 6, pp. 3792-3797, 2017.

[43] H. Wang, C. Wang, E. Matios, and W. Li, "Critical role of ultrathin graphene films with tunable thickness in enabling highly stable sodium metal anodes," Nano Letters, vol. 17, no. 11, pp. 6808-6815, 2017.

[44] A. Wang, X. Hu, H. Tang et al., "Processable and moldable sodium-metal anodes," Angewandte Chemie, vol. 129, no. 39, pp. 12083-12088, 2017.

[45] Y. Lee, J. Lee, J. Lee et al., "Fluoroethylene carbonate-based electrolyte with $1 \mathrm{M}$ sodium bis(fluorosulfonyl)imide enables high-performance sodium metal electrodes," ACS Applied Materials \& Interfaces, vol. 10, no. 17, pp. 15270-15280, 2018.

[46] Z. W. Seh, J. Sun, Y. Sun, and Y. Cui, "A highly reversible room-temperature sodium metal anode," ACS Central Science, vol. 1, no. 8, pp. 449-455, 2015.

[47] L. Liu, X. Qi, S. Yin et al., "In situ formation of a stable interface in solid-state batteries," ACS Energy Letters, vol. 4, no. 7, pp. 1650-1657, 2019. 